\title{
The effect of particle aspect ratio on spatially and angularly resolved vis-NIR spectroscopy of suspensions
}

\author{
Daria Stoliarskaia, Kelly Thomson, Leo Lue, and Yi-chieh Chen \\ Department of Chemical and Process Engineering, University of Strathclyde, James Weir \\ Building, 75 Montrose St, Glasgow G1 1XJ, UK
}

\begin{abstract}
The particle characteristics in a suspension affect the performance and quality of the end product of many chemical industries. The shape of the suspended particles can be influenced by changes in the manufacturing process conditions. Thus, there is a need for a robust method for continuous monitoring of particle characteristics through the process. This study investigates the feasibility of using spatially and angularly resolved diffuse reflectance measurements as a method of determining particle shape. A forward calculation was developed using the discrete dipole approximation to estimate optical properties of the single particle and the diffuse approximation for the reflectance of the particle suspension. The method was used to study aqueous suspensions of randomly-oriented polystyrene ellipsoids. Our objectives were to determine and elucidate the contribution of aspect ratio on optical measurement in vis-NIR spectra. The results suggest that the method is suitable for determining particle shape for suspensions where the particle and the solvent have significantly different optical properties. For these systems, the study suggests that diffusion reflectance measurements can be developed into an in-line method for particle shape determination
\end{abstract}

Keywords: Diffuse reflectance, diffuse approximation, DDSCAT, aspect ratio

\section{INTRODUCTION}

Today, there is a growing number of processes which require techniques for real-time characterisation of particle shape changes. ${ }^{1}$ The particle morphology (e.g., size, shape) and concentration of a suspension often critically dictate the performance of a formulation in many areas, such as in the chemical or pharmaceutical industry, and therefore need to be closely controlled. So far, most analytical approaches describe the particles with different geometries using the equivalent spherical diameter of the particle. ${ }^{2}$ Despite the practicality of these methods, overlooking the particle shape is known to cause difficulties to the flow of the material, homogeneity and performance of the end product. ${ }^{3-5}$ In addition, it can noticeably affect the quality of the estimation of other parameters, like particle size,${ }^{6}$ even when the particle shape parameters (e.g., roundness and aspect ratio) lie very close to spherical particle values.

The most accurate techniques in this field are based on image analysis to spot the most subtle differences in particle shapes. The methods often involve manual/semi-automatic image processing, where sampling preparation could be required. ${ }^{7}$ Both negatively affect the analysis speed. High-speed methods, on the other hand, often lack the accuracy due to a varying degree of noise associated with the images from the high-speed cameras. ${ }^{8}$ As an alternative, optical measurements are known to provide fast and non-invasive methods for characterising physical and chemical properties of materials. Although optical properties have been reported regarding their sensitivity to particle shape, optical measurement instruments have only made limited progress in identifying these effects. ${ }^{9,10}$ Depending on the particular physical measurement, limitations arise due to multiple scattering interruption for laser diffraction, or the indirect information from the chord length distribution for laser measurement. ${ }^{11,12}$

Further author information: (Send correspondence to D.S.)

D.S.: E-mail: daria.stoliarskaia@strath.ac.uk

L.L.: E-mail: leo.lue@strath.ac.uk, Telephone: +44 (0)141 5482470

Y.-C.C.: E-mail: yichieh.chen@strath.ac.uk, Telephone: +44 (0)141 5485304 
Such shortcomings can be addressed by combining an optical theory capable of accounting for multiple scattering and an instrument capable of performing such measurements in real-time. This requires a good understanding of light-particle interaction under multiple scattering conditions and the connection to the shape of the particles. To achieve this, the discrete dipole approximation (DDA) ${ }^{13}$ was employed in tandem with the diffuse approximation ${ }^{14}$ to simulate the optical response of a recently developed spatially and angularly resolved diffuse reflectance measurements (SAR-DRM) probe. ${ }^{15}$ The following work studies the effect of the variation in particle shape on the simulated reflectance. In order to localise the changes caused exclusively by particle shape, the suspensions are assumed to consist of randomly-oriented particles of a constant volume, hence those suspensions differ only in the ratio of length to width (aspect ratio) of the particles. In this study, the results of the modelled SAR-DRM spectra for the suspensions are reported.

The remainder of this paper is organised as follows. The next section gives an overview of the methods used to calculate the single particle optical properties and the diffuse reflectance of particle suspensions. Then the change in the diffusion reflectance of a suspension with the shape of its constituent particles is examined. This will gauge the feasibility for using the diffuse reflectance to determine particle shape. This is followed by a comparison of the results obtained with a reference calculation of the spherical particles. The results are analysed in the context of optical instrument design and multi-wavelength analysis. Finally, the key findings of the paper are summarized and directions for future work are considered in Section 4.

\section{METHODOLOGY}

\subsection{Calculation of bulk optical properties}

The first step of describing light-particle interaction is the calculation of the optical properties of a single particle. This study used the DDA method, which has been implemented in the open source software package Discrete Dipole SCATtering (DDSCAT), developed by Draine and Flatau. ${ }^{13}$ It is based on the straightforward idea of splitting the scattering body into small spheres of equal size, which is comparable to the size of the radiating dipole. Those dipoles are then placed in the lattice structure to form the desired shape of the particle. In addition to the shape, DDSCAT should be supplied with material properties, such as densities and complex refractive indexes.

The pre-packaged ellipsoid geometry within DDSCAT was used with most of the settings left at their default values with the exception of the orientation of the particle, the optical properties of the particle and the medium, and the wavelength range. In the suspension, the particles are randomly-oriented within the surrounding medium. Hence, the results of the DDSCAT simulations were averaged over 27 orientations with respect to the polarisation vector of the incident light. The calculations were repeated for 35 wavelengths in the range $450-1000 \mathrm{~nm}$. Properties at intermediate wavelengths were estimated using a cubic spline. ${ }^{16}$

For a single particle with volume $V_{p}$, DDSCAT provides the scattering $\sigma_{s}$ and absorption $\sigma_{a}$ cross-sections of the light, as well as the average scattering angle $\operatorname{cosine}\langle\cos \theta\rangle$ (also known as the asymmetry parameter $g$ ). For a collection of particles of the same size, these parameters are proportional to the concentration of the material in the system, which means, to obtain the bulk coefficients $\mu_{s}$ and $\mu_{a}$ for a monodisperse collection of particles, the cross-sections only require to be multiplied by a number of particles $N_{p} .{ }^{17}$ The asymmetry parameter remains the same in this case as it is independent of the number of particles. The end form of bulk optical properties can be expressed as follows:

$$
\mu_{s}^{\prime}(\lambda)=\left(\sigma_{s}(\lambda) N_{p}\right)(1-g) \quad \text { and } \quad \mu_{a}(\lambda)=\sigma_{a}(\lambda) N_{p}+\frac{4 \pi n_{\text {imag }}}{\lambda} \phi_{w}
$$

where $\mu_{s}^{\prime}(\lambda)$ is the reduced scattering coefficient that combines the scattering coefficient with the directionality of the scattered light, and $\mu_{a}(\lambda)$ combines contributions from both material and surrounding medium through volume fraction $\phi_{w}$ and imaginary part of complex refractive index $\left(n_{\mathrm{imag}}\right)$ of water. The number of particle per unit volume $N_{p}$ is derived from the mass fraction $C_{p}$, and the density of the particle and media $\left(\rho_{p}\right.$ and $\left.\rho_{w}\right)$ as:

$$
N_{p}=\frac{\rho_{w}}{\left(\rho_{w}+\rho_{p} / C_{p}-\rho_{p}\right) V_{p}}
$$




\subsection{Calculation of SAR-DRM spectra}

The correction for the number of particles in the system, presented in Equation 1, does not reflect the lightparticle interaction in the turbid medium accurately because such system exceeds the single scattering regime. The behaviour of the light which undergoes multiple scattering can be described using the radiative transport equation (RTE). ${ }^{18}$ However, numerically solving the RTE is computationally intensive, and, therefore, the diffuse approximation to RTE is developed to offer an alternative. ${ }^{14,19}$

The diffuse approximation employs the bulk optical properties in the form of a reduced scattering coefficient and a scattering coefficient, described in the previous section, as well as parameters related to the measurement instrument. Our model is based on the specifications of the spatially and angularly resolved diffuse reflectance measurements (SAR-DRM) probe used in the recent work by Chen and Thennadil. ${ }^{15}$ The chosen probe design has a number of receiving fibres, placed in a cross-setting at $0.3 \mathrm{~mm}$ from each other, forming a range of the $\mathrm{S}-\mathrm{R}$ distances. The source fibres are placed in the centre of the probe (normal emittance), and at the corners of the receiving fibres for angular light sources $\left(30^{\circ}\right.$ and $\left.45^{\circ}\right)$. Together, they form four different source-to-receiver distances: $0.3,0.6,0.9$ and $1.2 \mathrm{~mm}$. The diffuse approximation performance and the comparison of the results with SAR-DRM have been discussed in more detail by Thomson et al. ${ }^{20}$

\subsection{Materials}

To examine the impact of the alteration of particle form on the light scattering behaviour, the simulations were performed for particles of constant volume but different shapes. The measure of non-sphericity was defined as the ratio between the longest $(\mathrm{C})$ and minor $(\mathrm{A}$ and $\mathrm{B})$ semi-axis, the aspect ratio $(\mathrm{AR})$. This work studied prolate ellipsoids, one of the most common geometries, with three different aspect ratios: 1:1 (sphere), 3:1, and 8:1. These aspect ratios were converted into DDSCAT shape inputs with a dipole spacing set to 0.04A. Figure 1 illustrates the DDSCAT models of each type of particle studied, the dimensions of their semi-axes $(A, B$ and $C$ ), and the number of dipoles $N$ used for each of the particles. To keep the volume constant, semi-axes $A$ and $B$ have to decrease along with the increase of the semi-axis $C$.

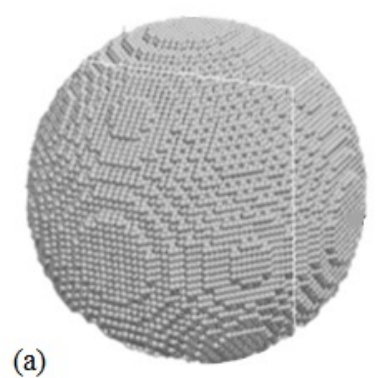

(a)

$$
\begin{gathered}
\mathbf{A R}=\mathbf{1 : 1} \\
\mathrm{A}=\mathrm{B}=250 \mathrm{~nm} \\
\mathrm{C}=250 \mathrm{~nm} \\
N=65,000
\end{gathered}
$$

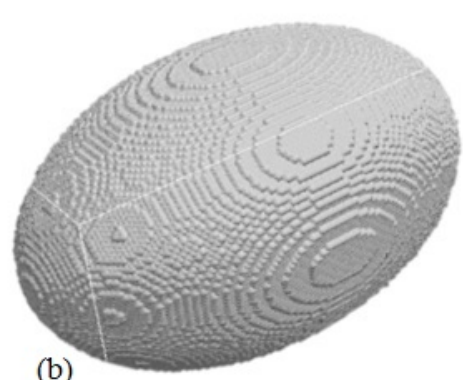

(b)

$$
\begin{gathered}
\mathbf{A R}=\mathbf{3 : 1} \\
\mathrm{A}=\mathrm{B}=176 \mathrm{~nm} \\
\mathrm{C}=500 \mathrm{~nm} \\
N=206,720
\end{gathered}
$$

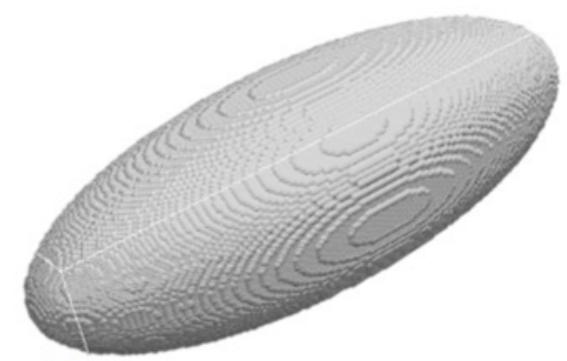

(c)

$$
\begin{gathered}
\mathbf{A R}=\mathbf{8 : 1} \\
\mathrm{A}=\mathrm{B}=125 \mathrm{~nm} \\
\mathrm{C}=1000 \mathrm{~nm} \\
N=520,000
\end{gathered}
$$

Figure 1: Polystyrene particles simulated by DDSCAT: (a) sphere, (b) prolate ellipsoid (AR = 3:1), and (c) prolate elliptical $(\mathrm{AR}=8: 1)$.

The colloidal suspensions were assume to be composed of monodisperse particles with $5 \%$ solid loading to ensure conditions that the conditions for diffuse scattering are met. The particle volume fraction remained constant across the samples, as the aspect ratio of the particles was varied. The material of the suspension was chosen to be polystyrene in water; the complex refractive indexes across the studied wavelength range were taken from the work of Sultanova et al. ${ }^{21}$ and Hale and Querry, ${ }^{22}$ respectively. 


\section{RESULTS AND DISCUSSION}

To gain an understanding of influence of the aspect ratio to light-particle interactions, the analysis was carried out on the simulated bulk optical properties. Figure 2 presents the scattering coefficient, asymmetry parameter, and absorption coefficient, where each line corresponds to a suspension with particles of a specified aspect ratio $(\mathrm{AR})$.
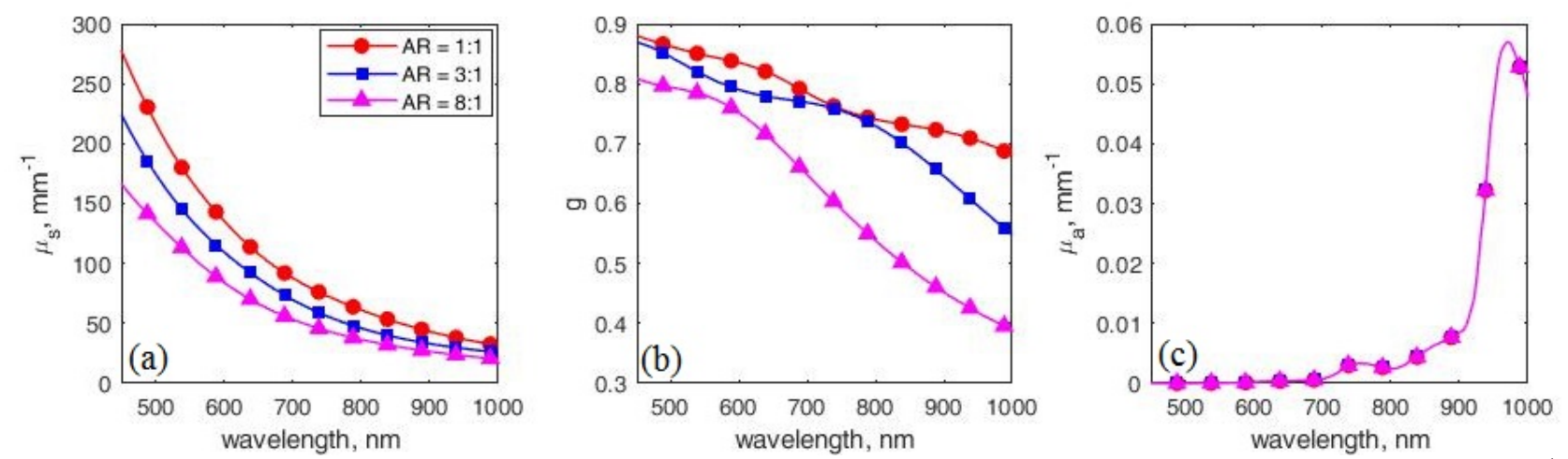

Figure 2: Bulk optical properties for polystyrene colloid suspensions for different particle aspect ratio: (a) scattering coefficient $\mu_{s}$, (b) asymmetry parameter $g$, and (c) absorption coefficient, $\mu_{a}$

The particle shape predominantly affects the scattering coefficient and the asymmetry parameter, whereas the absorption coefficient remains unchanged. The trend of the effects, however, varies, dependent on properties investigated. The scattering coefficient becomes weaker with an increase in aspect ratio and the effect appears as the change in the magnitude between samples solely. The asymmetry parameter, in contrast, changes in a much more specific manner with the AR. While $g$ for a sphere slowly decreases with increasing wavelengths and shows two distinctive peaks at 650 and $900 \mathrm{~nm}$, the same parameter for $\mathrm{AR}=3: 1$ exhibits a single peak at $700 \mathrm{~nm}$, and the magnitude decreases more quickly than for a sphere. Notably, the asymmetry parameter for AR $=8: 1$ drastically decreases after $650 \mathrm{~nm}$ as the wavelength increases, compared to the other two aspect ratios. Overall, changes in AR lead to the sufficiently large, specific changes in the bulk optical properties to distinguish between different particle shapes. However, the effects appearing in the scattering coefficient are not exclusively due to the particle shape, as changes in the particle size have a similar effect on scattering. ${ }^{17}$ For the case where a change in $\mu_{s}$ is due to changes in particle size, the absorption coefficient is expected to vary simultaneously, as given in the Eq. (1). To distinguish between these effects, multiple optical properties should be simultaneously.
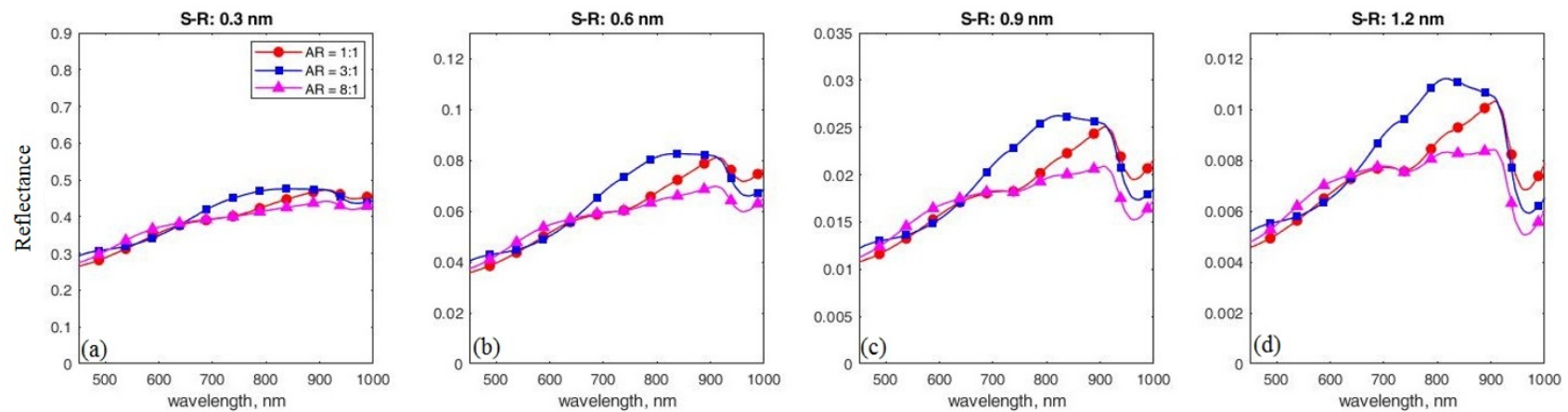

Figure 3: Diffuse reflectance simulated for various source-to-receiver (S-R) distances from normally emitted light. The symbols correspond to suspensions with particles of different aspect ratio: 1:1 (circles), 3:1 (squares), and $8: 1$ (triangles). (a)-(d) correspond to $\mathrm{S}-\mathrm{R}$ distance increase from 0.3 to $1.2 \mathrm{~mm}$ with $0.3 \mathrm{~mm}$ interval

The particle shape effect appears differently on the parameters studied for each of the wavelengths. The change in the length of particle significantly affects the asymmetry parameter at wavelengths between 700 to 
$1000 \mathrm{~nm}$; however, the effect on the scattering coefficient appears at lower wavelengths, $450-700 \mathrm{~nm}$. The strong wavelength dependence of the optical properties on the particle AR suggests that multiple wavelengths need to be analysed to properly deduce the particle shape.

In the next step, the optical properties were used to model SAR-DRM spectra. As previously described, the chosen spectroscopic technique emits light at different angles and collects the reflected signal at different S-R distances from the light source. Figure 3 shows the diffuse reflectance collected at various distances from the light source, S-R. Firstly, the simulated reflectance is compared using normal emittance for suspensions of particles with different aspect ratio. With a closer look at Figure 3(a), the overall SAR-DRM differs with the change of the aspect ratio, however, the visual appearance of the effect reduced compare to bulk optical properties observation. The order of the magnitude for samples has changed and the magnitude between them visibly reduced. With an increase of S-R distance, the intensity of the light has reduced proportionally. However, signal simulated for longer S-R distance has an increased separation between samples of different AR, clearly showing the effect of aspect ratio at the same wavelength range as observed in $g$ in Figure 2(b). The relative difference in intensity due to $\mathrm{AR}$ continues to increase with the increase of the S-R distance. It can be concluded that the difference in particle shape affects the SAR-DRM in the enough extend to consider inverting problem for experimental SAR-DRM spectra (i.e. to estimate the particle aspect ratio from measured spectra).
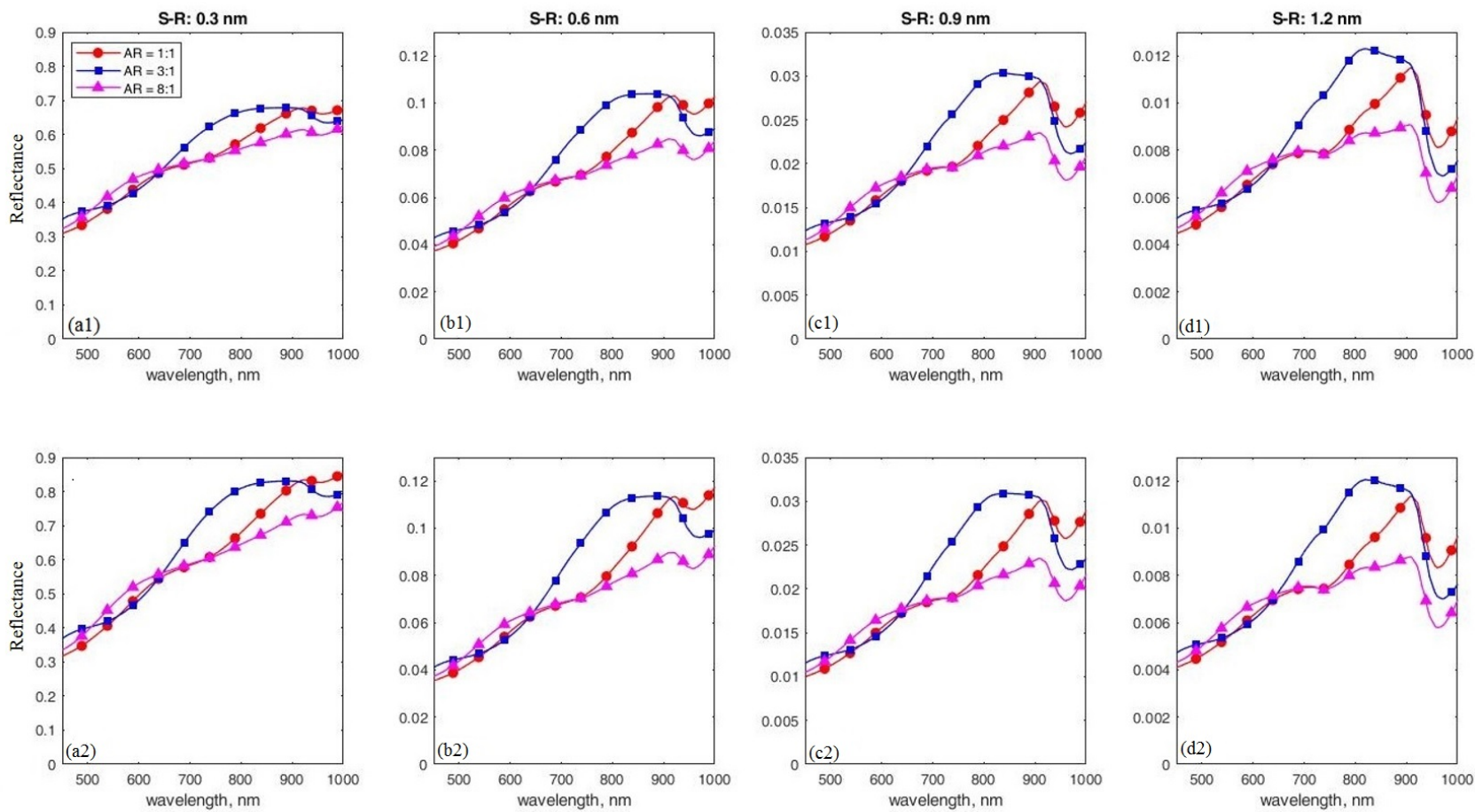

Figure 4: Diffuse reflectance collected at various source-to-receiver (S-R) distances from light emitted at a specified angle to the sample, (a1-d1): $30^{\circ},(\mathrm{a} 2-\mathrm{d} 2): 45^{\circ}$. Symbols correspond to suspensions with particles of different aspect ratio: 1:1 (circles), 3:1 (squares), and 8:1 (triangles). (a)-(d) correspond to S-R distance increase from 0.3 to $1.2 \mathrm{~mm}$ with $0.3 \mathrm{~mm}$ interval

Figure 3 shows a clear link between the effect of the aspect ratio and the spatial resolution of the spectra. Incorporating an additional light source produces two more datasets for analysis, which was illustrated in Figure 4. They were compared to normal emittance as well as the difference that angle itself $\left(30^{\circ}\right.$ and $\left.45^{\circ}\right)$ makes. Overall, the pattern of the spectra is similar to what was seen in Figure 3, however, the intensity of the reflectance is slightly higher and the relative difference in its slope has modified as well. Those changes indicate that suspensions with particles of different AR can be distinguished using signal collected from the angular light source at the smallest S-R distance, which was not possible when using normal incident light. The difference between the signal from $30^{\circ}$ and $45^{\circ}$ mostly appears in the relative change of the spectra slope between $750 \mathrm{~nm}$ 
and $900 \mathrm{~nm}$. The increase in the relative difference of the slope manifests in the more prominent difference between samples with a different aspect ratio. The particle AR has a stronger influence with increasing S-R, than with increasing angle of incidence of the light source, however, the intensity of the reflectance fades with an increase of S-R distance, which is a disadvantage at high signal-to-noise ratio. Using an angular source increases the strength of the signature signal arising from the particle AR and, consequently, is helpful in the estimation of particle AR.

In this study, the suspension was assumed to be isotropic (i.e. the particles are randomly oriented), and, therefore, the optical properties were obtained by averaging over a number of particle orientations. However, some of the orientations contribute to the scattering intensity and directionality to a greater extent than others. In order to obtain the most accurate estimate for the optical properties of the suspension, the DDSCAT calculations should be averaged over a sufficient number particle orientations. In addition, the diffuse approximation assumes that all photons collected in the reflectance measurements have undergone a large number of scattering events; this condition should be satisfied as long as the S-R distance is at least one path length. The measurements from furthest collecting fibre would be reflected in increasing path length through the sample, and, therefore, a sufficient distance must be chosen for the sensible inversion measurements from SAR-DRM.

Presence of such a strong effect of AR along with some specific changes between light collection settings substantiates the possibility to experimentally monitor particle aspect ratio in future studies. To invert the desired particle information successfully, one needs to verify that the solution to the inversion is unique. Therefore, the modelling should be extended to a number of aspects of the particle, not covered in this work. First, it is vital to investigate the contribution of material properties to the response seen. The SAR-DRM signal for a number of materials of different refractive index should be simulated in order to evaluate the degree that material has on the appearance of the aspect ratio effect. In addition, the sensitivity of the method to the different shapes should be considered by comparing the SAR-DRM calculation results for the different geometries of the same volume and/or surface area. The simulations presented should be analysed to identify the possibility of the presence of multiple minima to avoid ill-posed inversion problem of multiple solutions. By combining the above mentioned studies with the results presented, a suitable inversion methodology can be chosen for the inverting particle shape reliably.

\section{CONCLUSIONS}

In this work, the effect of the aspect ratio of the ellipsoidal particles on spatially and angularly resolved diffuse reflectance spectra was studied. To achieve this, randomly-oriented ellipsoid suspensions with the same volume with two different aspect ratios and their bulk optical properties were modelled using DDSCAT. The reflectance of the colloid suspensions was then computed using DA and the results were compared with the suspension of spherical particles of the same volume. The asymmetry parameter, $g$ and the scattering coefficient $\mu_{s}$ show the most prominent response to the change in aspect ratio. The effect of the aspect ratio is also manifested in the spatially and angularly resolved diffuse reflectance; it suggests that there is sufficient differentiation between samples for inversion. The probe design beneficially contributed to the observation of the effect as the effect of AR were seen the most strongly in SAR-DRM at large source-to-receiver distances. The aspect ratio influence was not constrained to a specific wavelength but rather appeared in a large wavelength range between $700-1000 \mathrm{~nm}$, which emphasizes the importance of multi-wavelength analysis.

There are a number of aspects that have to be clarified in future work. First of all, polystyrene is a highly scattering material and scattering properties of other materials might not show the influence of the aspect ratio influence to the same degree. Another point of interest is the similarity of the effect of particle AR in relation to the effects of other suspension parameters (e.g., particle size, concentration, etc.) on SAR-DRM spectra. This is crucial to understanding whether the proposed method would be useful in extracting particle shape information from SAR-DRM measurements.

\section{ACKNOWLEDGMENTS}

Authors thank the University of Strathclyde and an industrial partner for support of this project. 


\section{REFERENCES}

[1] Cruz, P., Rocha, F., and Ferreira, A., "Effect of operating conditions on batch and continuous paracetamol crystallization in an oscillatory flow mesoreactor," CrystEngComm 18, 9113-9121 (Royal Society of Chemistry 2016).

[2] Jennings, B. and Parslow, K., "Particle size measurement: The equivalent spherical diameter," Proceedings of the Royal Society of London. A. Mathematical and Physical Sciences 419(1856), 137-149 (1988).

[3] Chan, H.-K., "What is the role of particle morphology in pharmaceutical powder aerosols?," Expert opinion on drug delivery 5(8), 909-914 (2008).

[4] Ridgway, K. and Rupp, R., "The mixing of powder layers on a chute: the effect of particle size and shape," Powder Technology 4(4), 195-202 (1971).

[5] Fatah, N., "Study and comparison of micronic and nanometric powders: Analysis of physical, flow and interparticle properties of powders," Powder Technology 190(1), 41-47 (2009).

[6] Naito, M., Hayakawa, O., Nakahira, K., Mori, H., and Tsubaki, J., "Effect of particle shape on the particle size distribution measured with commercial equipment," Powder Technology 100(52-60) (1998).

[7] Pons, M.-N. and Dodds, J., "Particle shape characterization by image analysis," Progress in Filtration and Separation , 609-636 (2015).

[8] Anda, D., Calderon, J., Wang, X., and Roberts, K., "Multi-scale segmentation image analysis for the inprocess monitoring of particle shape with batch crystallisers," Chemical Engineering Science 60(4), 10531065 (2005).

[9] Kelly, K. L., Coronado, E., Zhao, L. L., and Schatz, G. C., "The optical properties of metal nanoparticles: the influence of size, shape, and dielectric environment," The Journal of Physical Chemistry, ACS Publications 107 (2003).

[10] I.Mishchenko, M., D.Travis, L., and W.Mackowski, D., "T-matrix computations of light scattering by nonspherical particles: A review," Journal of Quantitative Spectroscopy and Radiative Transfer 55(16), 3073-3077 (1996).

[11] Ma, Z., Merkus, H. G., and Scarlett, B., "Extending laser diffraction for particle shape characterization: technical aspects and application," Powder Technology 118(1-2), 180-187 (2001).

[12] Kempkes, M., Eggers, J., and Mazzotti, M., "Measurement of particle size and shape by fbrm and in situ microscopy," Chemical Engineering Science 63(19), 4656-4675 (2008).

[13] Draine, B. T. and Flatau, P. J., "User guide for the discrete dipole approximation code ddscat 7.3," arXiv preprint arXiv:1305.6497 (2013).

[14] Farrell, T. J., Patterson, M. S., and Wilson, B., "A diffusion theory model of spatially resolved, steadystate diffuse reflectance for the noninvasive determination of tissue optical properties in vivo," Medical physics 19(4), 879-888 (1992).

[15] Chen, Y.-C., Foo, D., Dehanov, N., and Thennadil, S. N., "Spatially and angularly resolved spectroscopy for in-situ estimation of concentration and particle size in colloidal suspensions," Analytical and bioanalytical chemistry 409(30), 6975-6988 (2017).

[16] Jones, E., Oliphant, T., Peterson, P., et al., "SciPy: Open source scientific tools for Python," (2011-).

[17] Bohren, C. F. and Huffman, D. R., [Absorption and Scattering of Light by Small Particles], no. ISBN:9780471293408, WILEYVCH Verlag GmbH \& Co. KGaA (December 2007).

[18] Ishimaru, A., [Wave propagation and scattering in random media], New York: Academic press (1978).

[19] Lin, S.-P., Wang, L., Jacques, S. L., and Tittel, F. K., "Measurement of tissue optical properties by the use of oblique-incidence optical fiber reflectometry," Applied Optics 36(1), 136-143 (1997).

[20] Thomson, K., Stoliarskaia, D., Tiernan-Vandermotten, S., Lue, L., and Chen, Y.-C., "Simulation of diffuse reflectance for characterisation of particle suspensions," Optical Diagnostics and Sensing XVII: Toward Point-of-Care Diagnostics. Coté, G. L. (ed.). Bellingham, Washington 10072, 5 (March 2017).

[21] Sultanova, N., Kasarova, S., and Nikolov, I., "Dispersion properties of optical polymers," Acta Physica Polonica-Series A General Physics 116(4), 585 (2009).

[22] Hale, G. M. and Querry, M. R., "Optical constants of water in the 200-nm to 200- $\mu$ m wavelength region," Applied Optics (3), 555-563 (1973). 